\title{
¿Cómo recomendar calzado deportivo a pacientes que practican running? Desde la evidencia científica a la experiencia clínica
}

\author{
How to give advise on footwear to running patients? From scientific evidence to clinical \\ experience
}

Manuel Mosqueira Ourens

Clínica del Pie Factor Biomecánico. A Coruña, España. Grupo de Investigación ABIDOR, Universidad Católica San Antonio de Murcia (UCAM), España

\section{Palabras clave:}

Correr, calzado deportivo, zapatillas de correr, asesoramiento, pronación, amortiguación, comodidad.

\section{Resumen}

Practicar deporte tiene una influencia positiva en la condición física de una persona, así como para reducir la incidencia de obesidad, enfermedades cardiovasculares y muchos otros problemas de salud crónicos. Correr es una de las formas más populares de actividad física, ya que es una actividad humana natural y accesible para casi toda la población. En los últimos 50 años la popularidad de la carrera a distancia ha aumentado debido a su gran accesibilidad y a la promoción del deporte desde el ámbito de la salud. Sin embargo, correr causa altas tasas de lesiones. La etiología es multifactorial, incluyendo factores extrínsecos como la superficie, el sobrentrenamiento, el nivel del corredor o la especialidad deportiva y los factores intrínsecos, como el nivel de condición física, el historial de lesiones, la fatiga y las adaptaciones durante la carrera, la biomecánica y las zapatillas de running.

El diseño del calzado para correr ha cambiado en los últimos cincuenta años con los objetivos de prevenir lesiones relacionadas con el running y mejorar el rendimiento. Las zapatillas para correr deben seleccionarse después de una evaluación cuidadosa y exhaustiva. Este artículo de actualización evalúa las características y métodos para recomendar zapatillas a los corredores. El objetivo es proporcionar una visión amplia, clara y objetiva de estas herramientas y ayudar a los podólogos en sus clínicas a la hora de recomendar zapatillas de running.

\begin{abstract}
Doing sports has a positive influence on a person's physical condition as well as for reducing the incidence of obesity, cardiovascular disease, and many other chronic health problems. Running is one of the most popular forms of physical activity as it is a natural human activity and accessible to almost everyone. Within the last five decades the popularity of distance running has increased due to its easy accessibility and sport promotion in general. However, running causes high rates of injuries. The multifactorial etiology, including extrinsic factors such as the surface, overtraining, skill level, the level of competence and intrinsic factors such as the level of physical condition, injury medical records, disturbances during the race, running biomechanics and running shoes.

Running footwear design has changed over the past fifty years in order to prevent running-related injuries and improve performance. Running shoes should be selected after a thorough and careful evaluation. This update article evaluated the characteristics and methods for giving useful advice on footwear to runners. The aim is to provide a broad, clear and objective view on this tools and help podiatric clinicians when recommending running shoes.
\end{abstract}




\section{INTRODUCCIÓN}

Correr (i.e. running) es una de las actividades deportivas más populares, ya que es una actividad natural del ser humano ${ }^{1}$ y accesible para casi todo el mundo. Tiene muchos beneficios positivos para la salud y puede contribuir a la prevención de numerosos problemas crónicos relacionados con la inactividad. Sin embargo, la prevalencia de lesiones en los miembros inferiores como consecuencia de la práctica del running sigue en aumento ${ }^{2}$, y aunque su etiología es claramente multifactorial ${ }^{3}$, el calzado deportivo es un factor clave a tener en cuenta. Es lógico considerar que las zapatillas de running juegan un papel fundamental, ya que generalmente es la única equipación necesaria para correr. Por ello, gran parte del éxito en el manejo de las lesiones en corredores pasa por la importancia de conocer el calzado deportivo ${ }^{4}$.

Es evidente que las zapatillas de running actuales son el resultado de investigación e innovación, y su cambio es constante a lo largo de los años con el objetivo de mejorar el rendimiento e influir en la biomecánica, así como ayudar a prevenir lesiones. Sin embargo, y a pesar del desarrollo tecnológico aplicado al calzado deportivo, hasta el $79 \%$ de los corredores se siguen lesionando en la actualidad ${ }^{5,6}$.

Tal y como indica Lieberman 7 , todos los corredores, incluidos los atletas profesionales, corrían descalzos o con un calzado minimalista hasta que en 1970 se inventaron las zapatillas de running modernas, que incorporaban amortiguación en el talón, soporte en el arco plantar y suelas más gruesas con el objetivo de aportar protección y atenuar el impacto ${ }^{8}$. Sin embargo, actualmente el calzado se diseña con suelas más finas para promover un patrón de apoyo más natural que, al mismo tiempo, su mecanismo de ejecución proteja al pie y al resto de estructuras de la superficie $e^{8,9}$. La teoría del profesor Lieberman de que el cuerpo humano estaría preparado para correr descalzo, y que incluso desde una perspectiva evolutiva correr descalzo es tan natural como caminar descalzo, se ve apoyado por estas investigaciones. Por ello, tal vez la cuestión sea que el pie está desadaptado a correr descalzo sobre las superficies actuales y también que el "corredor tipo" ha cambiado ${ }^{10}$, puesto que cada vez se incrementa más el número de corredores aficionados, sobre todo sin preparación previa, y proporcionalmente el número de lesiones también aumenta.

Los principales objetivos de las zapatillas de running siempre han sido los mismos a lo largo de la historia: proporcionar amortiguación y mejorar el rendimiento. El rendimiento es un parámetro que, si lo analizamos en función de las marcas de carreras de larga distancia como la maratón, podemos observar que se ha producido una clara y notoria disminución de los tiempos desde los primeros juegos olímpicos modernos de la historia hasta los pasados de Río de Janeiro ${ }^{11}$. Aunque si buscamos una relación entre marcas y zapatillas de running, deberíamos añadir una variable importante como es el corredor/a (principalmente la raza). Por ello es importante plantearse la pregunta: ¿la zapatilla hace al corredor?, o ¿es el corredor el que hace a la zapatilla?
Realmente estamos ante una compleja interacción multivariable que engloba el tipo de corredor, su anatomía (antropometría, biomecánica, etc.), técnica de carrera, historial de lesiones, métodos y carga de entrenamiento, superficie y, por supuesto, su calzado deportivo. Han sido varias las investigaciones que han intentado crear herramientas de asesoramiento en calzado ${ }^{12-15}$ y se han descrito hasta un total de 15 métodos distintos para asesorar hasta 28 características $^{16}$. Los beneficios de estos criterios para evaluar las características del calzado son evidentes, sin embargo, no está claro si hay más herramientas disponibles, cuáles se están utilizando y sobre todo si son relevantes, por ejemplo, en la consulta diaria de podología.

Dado que las zapatillas de running pueden afectar sustancialmente en la frecuencia de lesiones ${ }^{17}$, y que es habitual que los podólogos recomienden determinadas marcas y modelos de calzado deportivo a los pacientes para prevenir y/o tratar las lesiones, además de una detallada anamnesis y un examen clínico exhaustivo, es necesario evaluar en base a qué criterios y herramientas con evidencia científica y/o clínica se deben realizar estas recomendaciones sobre el calzado de running.

El objetivo del presente artículo consiste en dar una visión sobre el calzado deportivo en el running, la evolución de sus características principales y en base a qué parámetros se debe fundamentar su recomendación.

\section{EVALUACIÓN BIOMECÁNICA}

La recomendación o prescripción de calzado deportivo se ha centrado históricamente en factores relacionados con la amortiguación, el control de la pronación, la actividad muscular y el control postural, así como el rendimiento deportivo anteriormente mencionado. Sin embargo, el parámetro más habitual es lo denominado como "biomecánica del corredor". Pero, ¿qué debemos evaluar en el binomio corredor-calzado desde un punto de vista biomecánico?

El objetivo principal es el de identificar factores de riesgo biomecánico y limitaciones/alteraciones funcionales que predispongan a la lesión en el corredor/a. Además de las valoraciones en camilla o en bipedestación que se llevan a cabo habitualmente en la clínica diaria, la evidencia científica pone de manifiesto la importancia de evaluar los movimientos del corredor en test lo más funcionales y dinámicos (con mayor validez que las pruebas en descarga), tanto en condición descalza como posteriormente, realizar de nuevo los mismos test y comparar los resultados en condición calzada ${ }^{4}$. Probablemente es correcto indicar que faltan pautas clínicas sobre la importancia y los usos de las herramientas de evaluación del calzado en el binomio corredor-calzado, así como entender posibles diferencias en situaciones en las que el paciente realiza tareas similares en condiciones descalza y calzada. La realización de estas pruebas funcionales genera patrones de movimiento y activaciones musculares 
de todo el cuerpo, y principalmente del aparato locomotor, y pueden identificar déficits de movilidad o de fuerza que no son obvias en el examen previo a través de las pruebas más habituales o comunes en la valoración clínica y podológica del corredor/a.

Uno de los test que reflejan y reproducen la importancia de lo indicado en el párrafo anterior es el single leg heel raise o elevación con una sola pierna. Este test es una prueba clínica muy habitual para evaluar la función del pie, el tobillo, la fuerza del tríceps sural y tendón de Aquiles, así como la fatiga, la resistencia y el equilibrio ${ }^{18,19}$. También se debe utilizar al evaluar al corredor/a reproduciendo este test en condición descalza y calzada. La realización de este test en clínica nos permite evaluar el efecto de posibles desgastes en el calzado (zona posterior lateral o medial) y de la compresión de la mediasuela, y puede proporcionar información sobre cómo el calzado altera la retroalimentación neuromuscular ${ }^{20}$.

La realización de otros test, como el single leg squat ${ }^{21}$ o el single leg hopping ${ }^{22}$, son recomendables en corredores, son útiles para valorar posibles desequilibrios de fuerza en el miembro inferior y deben realizarse también en las dos condiciones (i. e. descalza y calzada).

Además debemos tener presente, como indican diferentes autores ${ }^{23}$, que en cualquier deportista que entrene y compita predominantemente corriendo, cualquier evaluación biomecánica y/o podológica debe basarse en el análisis de la carrera simulando las condiciones habituales destacando, principalmente, el calzado. Probablemente sea necesario y fundamental evaluar el calzado de running del paciente en cualquier estudio biomecánico en búsqueda de patrones asimétricos que pueden ser el resultado de diferencias en la valoración funcional del paciente cuando está calzado y descalzo ${ }^{14}$.

Otro de los aspectos a tener en cuenta en la valoración biomecánica es el tipo de contacto inicial $(\mathrm{Cl})$ que realiza el corredor/a en el momento de impactar con la superficie. Relacionado con el $\mathrm{Cl}$ es importante destacar que la fatiga influye claramente en la cinemática de carrera ${ }^{24} \mathrm{y}$, por tanto, en el patrón de $\mathrm{Cl}$, provocando una traslación de los $\mathrm{Cl}$ de metatarso y de la parte media del pie hacia el talón a medida que avanzan los kilómetros de la competición. Aunque en clínica, al igual que en estudios en laboratorio, no siempre se puede evaluar a los corredores en presencia de fatiga, lo cual puede reducir la validez de los resultados obtenidos, sí es necesario tenerla en cuenta a la hora de analizar posibles lesiones asociadas y también en la recomendación de zapatillas de running, ya que la fatiga es un componente crucial y casi omnipresente en la carrera ${ }^{25,26}$.

En consonancia con lo comentado anteriormente, los corredores de una maratón muestran mayoritariamente un $\mathrm{Cl}$ de talón ${ }^{27,28}$, y también se ha observado que a medida que se incrementa el rendimiento (i.e. mejor clasificación) los corredores presentan un menor $\mathrm{Cl}$ de talón y más con la parte media del pie o de metatarso.

Es importante tener en cuenta la experiencia del corredor/a, dado que aquellos pacientes que hayan comenzado a correr recientemente pueden ser más propensos a errores de entrenamiento y sobrecargas si no son monitorizadas por profesionales, como entrenadores y preparadores físicos.

En conclusión, parece que el patrón biomecánico de carrera relacionado con el pie (i.e., $\mathrm{Cl}$ ) está condicionado, entre otros factores, por la velocidad de carrera (i.e., rendimiento o nivel del atleta) y el nivel de fatiga experimentado por el atleta. Por ello es fundamental que, para comprender plenamente los riesgos y los beneficios de correr, sobre todo durante las primeras fases de la adaptación, y para la recomendación de calzado de running, entre otros, se requiere conocer los efectos de la fatiga.

La anamnesis es fundamental en todo tipo de pacientes y en corredores también. En estos casos, además de las cuestiones relacionadas con la salud, las médicas y las clínicas, es importante añadir una serie de preguntas como las indicadas en la Tabla I sobre aspectos relacionados con el entrenamiento, al igual que mantener una relación directa con el entrenador/a del corredor/a y aportar una valoración, control y seguimiento multidisciplinar.

A continuación se analizan los parámetros más utilizados para la supuesta prevención de lesiones a través de las zapatillas de running, como la amortiguación y la estabilidad. Históricamente se ha esgrimido que los materiales más blandos amortiguaban más, que la pronación era un factor de riesgo, y por lo tanto necesario el control de movimiento, y que, uniendo estos dos parámetros, se podrían prevenir lesiones en los corredores.

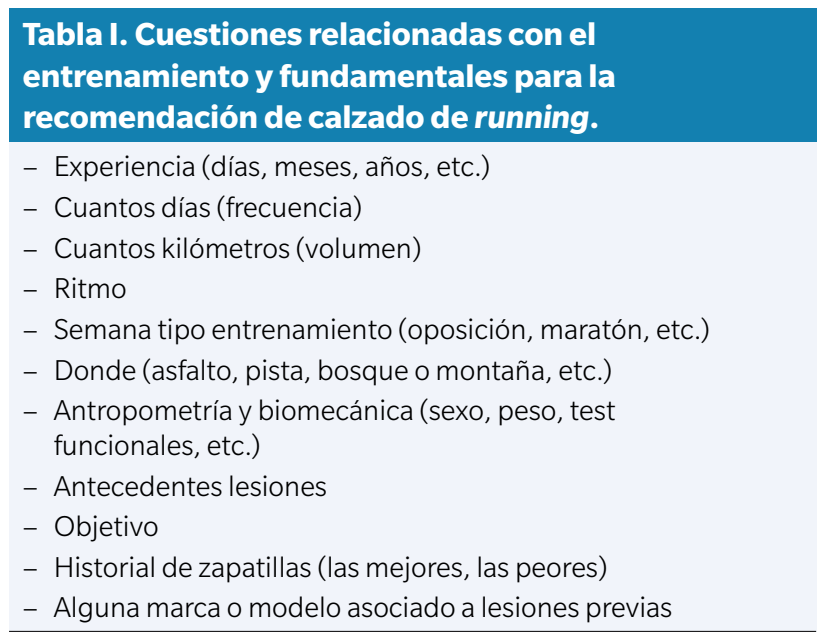

\section{AMORTIGUACIÓN}

A medida que las lesiones en el running se hicieron más frecuentes, las primeras teorías asociaban el riesgo de lesión al aumento de las fuerzas de impacto o de reacción del suelo. Esto derivó en el diseño y fabricación de zapatillas de running acolchadas con media suelas gruesas ${ }^{29}$. Sin embargo, 
y a pesar de que actualmente la tendencia es en la dirección opuesta y defiende que un apoyo más natural puede prevenir lesiones, las tasas de estas entre los corredores minimalistas y amortiguados son similares.

Del mismo modo, en los últimos años las investigaciones y publicaciones científicas indican que utilizar materiales más blandos no se traduce necesariamente en una mayor amortiguación, e incluso que las mediasuelas más blandas incrementan las fuerzas de reacción del suelo. Ejemplo de esto son los estudios que analizaron la amortiguación como una estrategia de prevención de lesiones, y sus resultados no mostraron una disminución significativa de la frecuencia de las lesiones al cambiar la dureza de la mediasuela ${ }^{30}$.

Es por ello fundamental tener en cuenta que un exceso de amortiguación en la zapatilla de running podría originar lesio$n^{31} s^{31}$ a umentar las fuerzas de reacción del suelo ${ }^{32}$ y definitivamente añadir más a la zapatilla no reduce el riesgo de lesiones en corredores ${ }^{30}$. A pesar de esto, la mayoría de los corredores creen que las zapatillas con grandes sistemas de amortiguación son eficaces a la hora de reducir el riesgo de lesiones en el running. Todo esto suponiendo que el aumento de las fuerzas de reacción del suelo sean un factor de riesgo en los corredores, dado que cada vez son más los estudios ${ }^{33,34}$ que discrepan y ponen en duda que sean culpables en el origen de las lesiones.

Sin embargo, y desde un punto de vista neuromecánico, las fuerzas de impacto contra el suelo durante el running servirían como señales de entrada ${ }^{35}$, que ayudan a producir un "ajuste muscular" al contacto del pie con el suelo (i.e. $\mathrm{Cl}$ ). Este ajuste ayuda a minimizar la vibración de los tejidos blandos y a reducir la carga de las articulaciones y los tendones. En otras palabras, aumentaría la amortiguación natural del cuerpo y reduciría el impacto del pie al contacto con el suelo. A esta teoría se la denomina "muscle tuning" y es tenida en consideración por las marcas de calzado de running en el diseño de sus zapatillas.

Cada marca deportiva tiene su sistema de amortiguación (Tabla II). Independientemente de estos, lo importante es que a medida que disminuye la capacidad de amortiguación de la zapatilla, los corredores sepan y puedan modificar sus patrones de apoyo y técnica de carrera para mantener las cargas externas constantes y controladas.

Relacionado con las estrategias de adaptación frente al desgaste del calzado, los estudios ${ }^{36}$ indican que no son modificadas por las diferentes tecnologías de amortiguación, por lo tanto se sugiere que los corredores deberían elegir el calzado de running por otras razones distintas a la tecnología de amortiguación.

\section{PRONACIÓN}

Para evitar lesiones relacionadas con el running, se ha prestado mucha atención, junto a las capacidades de absorción de impactos de la zapatilla, a los elementos de control de movimiento. Históricamente se ha recomendado comprar las zapatillas de running en función de parámetros como el grado de pronación ${ }^{37}$, sin embargo, la evidencia científica actual y los ensayos controlados aleatorizados no respaldan estas recomendaciones ${ }^{38,39}$.

A pesar de que la pronación es un movimiento activo y natural del pie para la adaptación y absorción de impactos durante la marcha y/o la carrera y la consecuente adaptación al terreno, siempre ha sido la principal variable y la más evaluada por los podólogos, y se ha asociado con las lesiones. Como consecuencia, cuando se comenzó a recopilar y a estudiar el riesgo de lesiones en los corredores, se consideró a la pronación, con poca evidencia científica al respecto, como una variable responsable del origen de las lesiones al correr y, a la vez, como importante para el diseño y fabricación del calzado de running. Sin embargo, estudios como el de Nielsen y colaboradores ${ }^{38}$ destacan en sus resultados y conclusiones que una posición pronada del pie (entre $7^{\circ}$ y $10^{\circ}$ ) es un factor preventivo y una ventaja con respecto a las lesiones en el running.

También es importante destacar los resultados de otro estudio ${ }^{39}$ donde se evidenció que los corredores con pies muy pronados y que usaron zapatillas con control de pronación se lesionaron más y estuvieron más días de baja que aquellos que, con el mismo tipo de pie, utilizaron zapatillas neutras. Esto nos puede hacer pensar que aquellos pacientes corredores con pies muy pronados requieren de cierto grado de libertad de movimiento para poder adaptarse a las superficies durante el contacto del pie con el suelo, y que un pie muy pronado tendría limitada su capacidad de absorber el impacto dado que es a través de la pronación como lo realiza. La aplicación clínica de esto sería plantearse la no utilización de calzado de running con grandes sistemas de control de pronación en aquellos pies muy pronados.

En vista de lo anterior, y de que no existe evidencia clara de que la pronación sea responsable de las lesiones en el running, la cuestión es determinar si el corredor prona de manera excesiva y si ese exceso puede ser lesivo. Para ello, las empresas y fabricantes de calzado de running han modificado sus sistemas se clasificación de las zapatillas eliminando el concepto de control de pronación por estabilidad; de esa manera se intenta transmitir la idea de que se busca mantener en un rango seguro la pronación, reduciendo la velocidad de este movimiento.

Tabla II. Actuales sistemas de amortiguación de las principales marcas de running.

\begin{tabular}{lllllllll} 
Marca & Asics & Brooks & Saucony & Mizuno & Nike & Adidas & New Balance & Hoka \\
\hline Sistema de amortiguación & Flytefoam & SuperDNA & Everun & Wave & ZoomX & Boost & FreshFoam & RMAT \\
\hline
\end{tabular}


Incluso el calzado de running con sistemas de medias suelas en la zona medial más duras, a menudo se degradan más rápido en la media suela lateral que es más blanda y este incremento de la asimetría puede contribuir a lesiones por inversión del tobillo, incrementar el estrés medial en la rodilla y las fuerzas de reacción del suelo ${ }^{40-42}$. Por este motivo los modelos actuales de prácticamente la totalidad de las marcas de calzado de running han incorporado elementos como las guías de control (se desarrollarán en posteriores apartados) y las mediasuelas abovedadas que abrazan al pie restándole protagonismo al incremento de la dureza de la mediasuela medial.

Todo esto nos debe hacer plantearnos que estamos orientando la prevención de lesiones en los parámetros equivocados, e incluso que estos parámetros pueden ser factores de riesgo en lugar de preventivos.

Es por ello que, en la actualidad, las marcas de calzado deportivo comienzan a plantear lo que se denomina como patrón preferido de movimiento y el filtro de confort como los verdaderos factores para la prevención de lesiones en corredores.

\section{PATRÓN PREFERIDO DE MOVIMIENTO}

Este concepto guarda similitudes con el efecto de las ortesis plantares y el concepto de realineación del pie, dado que: ¿es la congruencia articular del pie en posición neutra la óptima para la prevención de lesiones? La aplicación de la teoría de Root $^{43}$ en búsqueda de la posición neutra de la articulación subastragalina durante la fase de apoyo medio de la marcha con el objetivo de evitar compensaciones denominadas como "anormales" del pie durante dicha fase, y la realineación de la arquitectura del pie provocando cambios cinemáticos a través de las ortesis funcionales, ha sido y continúa siendo una teoría muy utilizada en el ámbito podológico. Sin embargo existen varias investigaciones mostrando que los cambios cinemáticos observados con el uso de las ortesis plantares son pequeños, débiles e inconsistentes ${ }^{44,45}$.

En el caso de las zapatillas de running la situación es la semejante y se puede explicar a través de un nuevo concepto definido por el profesor Benno Nigg en uno de sus artículos más recientes ${ }^{10}$ : "preferred movement pathway" o patrón preferido de movimiento.

La base de su teoría proviene de que la correlación entre la "alineación" del pie y la frecuencia de las lesiones es pequeña. Su explicación se fundamenta en que sí existen cambios entre correr descalzo y calzado, pero los que se producen en la trayectoria del movimiento en el calcáneo y la tibia son pequeños y no sistemáticos ${ }^{46,47}$, al igual que ocurre con las ortesis plantares, como se indicó anteriormente. Por ello es importante concluir que los cambios ocurren principalmente en el rango de movimiento, pero no en la trayectoria del movimiento.

Si lo citado anteriormente lo aplicamos a la correcta selección de calzado de running para nuestros pacientes, una adecuada zapatilla de correr sería aquella que permita al pie

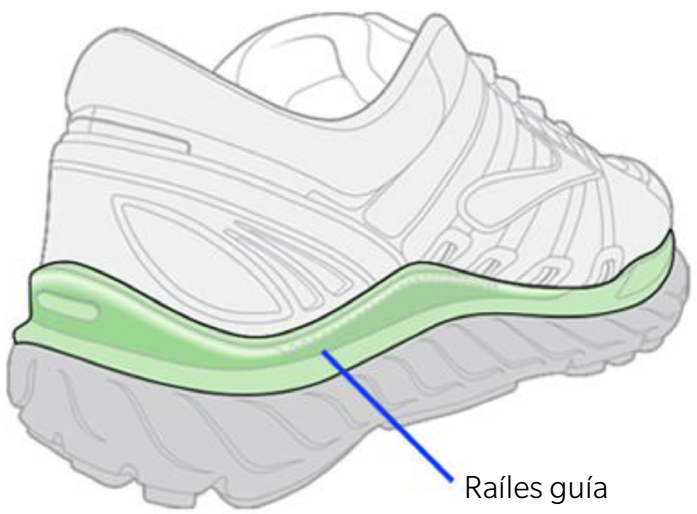

Figura 1. Sistema de control y estabilización a través de railes de guía (Brooks Sports, Inc.).

moverse en el patrón preferido de movimiento, teniendo presente la necesidad de limitar o acotar esa trayectoria de movimiento sin bloquearla. Este es el objetivo de las guías de control (guide rails) que prácticamente la totalidad de marcas de calzado de running han incorporado a sus modelos de zapatilla de running (Figura 1).

Lo que se intenta transmitir con el concepto de patrón preferido de movimiento es que no es posible controlar la cinemática del pie en gran medida y que los cambios que pueden producir las zapatillas de running están más relacionados con la activación muscular (muscle tuning) que con la realineación del esqueleto.

\section{CONFORT}

Es el parámetro más utilizado actualmente para recomendar calzado y zapatillas de running. No solo defendido por los estudios científicos ${ }^{48,49}$, sino también por las marcas de calzado deportivo y que muestran en sus campañas de marketing y publicidad. Aunque muchos corredores pueden identificar rápidamente qué zapatillas les resultan más cómodas, lo cierto es que el confort no es un parámetro fácil de cuantificar, por lo tanto... ¿Cómo evaluamos el confort de nuestros pacientes corredores?

Si le pedimos a un paciente que califique la comodidad de una zapatilla de running, no puede dar esa respuesta a menos que compare subjetivamente las sensaciones de ese calzado con las de otros que haya usado antes, y ese, ya es un parámetro a tener en cuenta no solo desde un punto de vista de elección, sino de descarte. Es decir, si un corredor nos indica que determinada marca o modelo de zapatilla le ha resultado cómoda, nuestro objetivo será buscar aquellas con características similares y testarlas en ese paciente. Es la teoría de "si algo funciona, no lo cambies", o al menos no en gran medida.

Otra medida o variable para evaluar el confort sería aqueIla que nos permitiese cuantificar la comodidad y determinar 


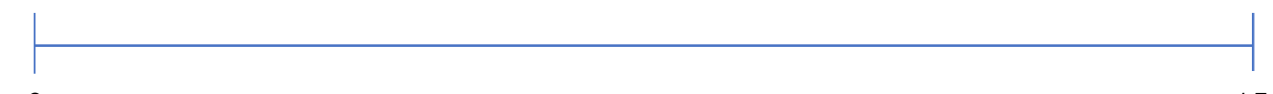

0

15

Nada cómodo

Lo más cómodo

Figura 2. Escala visual analógica para evaluar el confort.

\section{Tabla III.}

\begin{tabular}{ll} 
Parámetro a evaluar & Pregunta al paciente \\
\hline Nivel de amortiguación en general & ¿Le resultan amortiguadas estas zapatillas? \\
\hline Amortiguación en talón & ¿Ha notado demasiado impacto en el talón? \\
\hline Sujeción/adaptación en talón (cazoleta) & ¿Le confiere sujeción la parte posterior? \\
\hline Altura del arco longitudinal interno & ¿Le recoge bien el arco del pie? \\
\hline Ancho de la zapatilla & ¿Considera que se adaptan bien al ancho de su pie? \\
\hline Ancho del antepié & ¿Le aprietan o nota hormigueo con estas zapatillas? \\
\hline Longitud de la zapatilla & ¿Se adaptan bien a la longitud de su pie? \\
\hline
\end{tabular}

relaciones válidas entre esta y la estructura de las zapatillas, las características del corredor y sus variables biomecánicas ${ }^{12}$. Para ello podemos utilizar una escala visual analógica (VAS) (Figura 2) donde etiquetaríamos como "nada cómodo" (0 puntos de confort) a la izquierda, y en el extremo derecho "lo más cómodo" (15 puntos de confort).

Del mismo modo, y dado que hay otros aspectos que podrían influir en la comodidad, se incluirían preguntas específicas como las que figuran en la Tabla III.

En conclusión, si el calzado compromete la vía de movimiento preferida, aumentaría la carga y el aumento de la carga puede provocar lesiones ${ }^{10}$. Al contrario, si los corredores perciben que su calzado es cómodo, la incidencia de lesiones disminuye $\mathrm{e}^{49,50}$. Además, el calzado cómodo aumenta el rendimiento ${ }^{48}$ y reduce la variabilidad cinética durante la carrera ${ }^{51}$.

Determinar y cuantificar el confort es difícil. Sin embargo, y en base a la evidencia científica, clínica y comercial expuesta anteriormente, es un factor muy importante y se debe tener en cuenta cuando recomendamos calzado de running a nuestros pacientes.

\section{UTILIZACIÓN DE ORTESIS PLANTARES}

La duda de qué tipo de calzado deportivo de running es el más adecuado en función de si el paciente utiliza ortesis plantares es otro de las dudas habituales en consulta. Según la evidencia científica, esta decisión se apoya en dos parámetros: dureza y comodidad. Diferentes estudios han planteado que una ortesis (estándar o a medida) menos rígida podría reducir las lesiones, y son varios los que afirman que el 50-90 \% de los corredores que son tratados con ortesis plantares refieren una recuperación completa o una gran mejoría de sus patologías.
También es importante destacar y tener en cuenta, como se ha indicado anteriormente, que el efecto de realineación de las ortesis plantares no está claro y continúa siendo controvertido.

Relacionado con la comodidad, Muendermann y cols. ${ }^{49}$ incluyeron en su estudio, como único criterio de selección para las plantillas, la comodidad individual. Sus resultados mostraron que el grupo que escogió las plantillas que le resultaban más cómodas (independientemente de criterios podológicos o relacionados con la valoración clínica del paciente), tuvieron un $53 \%$ menos de lesiones que el grupo control. Por lo tanto, parece que la comodidad de las plantillas (ortesis plantares) es un factor importante para las lesiones, aspecto que como se ha podido evidenciar en el apartado anterior se extrapola al calzado de running.

Del mismo modo, la comodidad de las plantillas u ortesis plantares se ha asociado con varios factores, como la presión plantar, la sensibilidad del pie y la ya indicada alineación del pie y la pierna ${ }^{52,53}$. Ensayos clínicos aleatorizados encontraron que las ortesis plantares prefabricadas prescritas en función de la comodidad (en lugar del tipo de pie) reducen el dolor patelofemoral ${ }^{54,55}$.

Desde la experiencia del autor, y compartido con otros compañeros en situaciones habituales en consulta, el factor comodidad es fundamental: los pacientes que desde un primer momento no sienten cómodas sus ortesis plantares son claros candidatos a que tengamos que modificar nuestro tratamiento.

\section{OTROS FACTORES A TENER EN CUENTA}

El calzado de running desgastado induce movimientos compensatorios $^{36}$, reduce la capacidad de absorción de 
impactos ${ }^{56}$ y altera la estabilidad de la extremidad inferior ${ }^{57}$. Es importante tener en cuenta que la información a medida que se degrada la mediasuela del calzado de running los corredores pueden ver alterada la información sensorial que reciben a través del impacto y que la acumulación de entrenamiento o carga contribuyan a lesiones por sobreuso.

Es importante controlar la fatiga que pueden experimentar los materiales de la zapatilla de running (arrugas, dureza, etc.) pudiendo monitorizar este desgaste con durómetros destinados a tal objetivo. La zona del upper o tejidos de la zona superior de la zapatilla también deben de revisarse. Normalmente, a mayor peso del corredor mayor desgaste, al igual que si la superficie por la que habitualmente practica running es más abrasiva. El tipo de contacto inicial $(\mathrm{Cl})$ del pie con el suelo (talón, mediopié o metatarso) y mayores momentos pronadores/supinadores también deben de revisarse regularmente.

Se debe solicitar a los corredores que acudan a la clínica podológica con todas las zapatillas de running que utilicen actualmente (en numerosas ocasiones son varios modelos), así como si conservan las utilizadas anteriormente para valorar posibles desgastes, zonas de roce o presión, etc. Es importante determinar los meses o años y el número de kilómetros que tienen las zapatillas.

Relacionado con los parámetros indicados anteriormente, si el corredor indica que determinada marca o modelo le ha resultado cómodo, no se ha lesionado, y en general "le va bien", es recomendable no variar ni cambiar mucho las características de la nueva zapatilla, y es probable que busquemos algo similar y que aporte algún detalle extraído de la exploración biomecánica. Del mismo modo, pero en sentido contrario: si algo ha ido mal (sistema de amortiguación, dispositivos de control, etc.) tenerlo en cuenta para analizar las posibles razones en la exploración y, a priori, buscar otros modelos distintos a este.

El patrón de atado o la lazada en las zapatillas de correr también es un factor importante a tener en cuenta. Se produce un acoplamiento más firme del pie en una zapatilla con cordones que cubrieron hasta el último ojal superior, conduciendo a un uso más eficaz de las características de rodamiento del calzado deportivo y, probablemente, reduciendo el riesgo de lesiones de los miembros inferiores ${ }^{58}$. De igual modo, y teniendo en cuenta las conclusiones de otro estudio ${ }^{59}$, se debe controlar el uso de cordones elásticos dado que los corredores que los utilizan podrían tener un mayor pico de presión plantar en la zona lateral del antepié, así como una mayor pronación del retropié. Se debe tener en cuenta que, en general, los cordones pueden ayudar a los corredores a obtener un mejor ajuste del pie con la zapatilla, puesto que aumentan el confort percibido ${ }^{60}$ y ayudarían a prevenir lesiones, al permitir un mejor control de los factores antes mencionados.

También se recomienda que los corredores utilicen más de un par de zapatillas deportivas ${ }^{61}$, que varíen la distancia e incluyan otras actividades deportivas en su plan de entre- namiento ${ }^{30}$. Mejorar la movilidad, la estabilidad y la fuerza en los pies también ayudarían a los corredores a evitar lesiones?.

En este contexto de confort, es fundamental escuchar al corredor. Tal y como se ha propuesto, la anamnesis y entrevista personal es una parte importantísima dentro de la valoración global al corredor. También se deben de tener en cuenta la recomendación de otros profesionales como tiendas especializadas, entrenadores, etc., con el objetivo de aportar una visión lo más global y multidisciplinar posible al corredor.

\section{CONCLUSIONES}

El calzado deportivo de running ha ido evolucionando a lo largo de los años y es fundamental conocer estos avances para mejorar la recomendación y asesoramiento a nuestros pacientes.

Que una zapatilla de running tenga más amortiguación no implica ni se asocia a mejorar la capacidad para dispersar las fuerzas de reacción del suelo. Incluso el aumento de amortiguación a través de materiales más blandos puede alentar a los corredores a adoptar una técnica de carrera y un $\mathrm{Cl}$ menos eficiente y contactar con mayor impacto que las zapatillas con menos amortiguación.

La pronación es un movimiento natural y normal del pie al caminar y por supuesto también al correr. La pronación por sí sola no debería ser una razón para seleccionar una zapatilla de running; por ello el control de pronación en las zapatillas puede ser un elemento de riesgo en las lesiones por sobreuso en los corredores; cada vez está en mayor desuso y cada vez se emplea más el término estabilidad. En pies muy pronados o máximamente pronados, podría ser recomendable utilizar un calzado estable que permita pronar al pie consiguiendo una amortiguación más natural. Detener la pronación con materiales o densidades muy duras podría causar problemas y lesiones en los corredores.

Las lesiones producidas en el running son multifactoriales y están asociadas principalmente a parámetros de entrenamiento (entrenar mucho, muy rápido y sin descanso para asimilar las cargas), el sexo, la antropometría y biomecánica del corredor/a, así como las superficies y el calzado.

La valoración multidisciplinar, desde el podólogo, el entrenador y hasta la recomendación de la tienda especializada, proporcionan un mayor conocimiento y seguridad a la hora de indicar las zapatillas de running más adecuadas para los pacientes corredores.

Es fundamental escuchar y tener presente las sensaciones y preferencias del corredor a la hora de recomendar zapatillas de running. Conocer las características y los sistemas de amortiguación o de control de movimiento que haya utilizado y su experiencia con ellos (sobre todo si ha sido negativa), es fundamental para guiar el proceso de recomendación de calzado. El confort es un factor esencial a la hora de recomendar calzado y zapatillas de running. 
En base a lo indicado y referenciado anteriormente, para un paciente corredor/a es más importante cómo corra que el calzado que esté utilizando, pero también es esencial tener en cuenta que lo que lleva en sus pies, dado que puede afectar a cómo corre.

\section{CONFLICTO DE INTERESES}

Ninguno.

\section{FINANCIACIÓN}

Ninguna.

\section{BIBLIOGRAFÍA}

1. Bramble DM, Lieberman DE. Endurance running and the evolution of Homo. Nature. 2004;432(7015):345-52. DOI: 10.1038/nature03052.

2. Tschopp M, Brunner F. [Diseases and overuse injuries of the lower extremities in long distance runners]. Z Rheumatol. 2017;76(5):44350. DOI: 10.1007/s00393-017-0276-6.

3. Windt J, Gabbett TJ. How do training and competition workloads relate to injury? The workload-injury aetiology model. Br J Sports Med. 2017;51(5):428-35. DOI: 10.1136/bjsports-2016-096040.

4. Ramsey C, Lamb P, Ribeiro DC. Clinicians perceptions of footwear when assessing and managing patients with running-related injuries. Phys Ther Sport. 2020;42:9-15. DOI: 10.1016/j.ptsp.2019.12.005.

5. van der Worp MP, ten Haaf DSM, van Cingel R, de Wijer A, Nijhuis-van der Sanden MWG, Staal JB. Injuries in runners; a systematic review on risk factors and sex differences. PLoS One. 2015;10(2):e0114937. DOI: 10.1371/journal.pone.0114937.

6. Hulme A, Nielsen RO, Timpka T, Verhagen E, Finch C. Risk and Protective Factors for Middle- and Long-Distance Running-Related Injury. Sports Med. 2017;47(5):869-86. DOI: 10.1007/s40279-016-0636-4.

7. Lieberman D. What We Can Learn About Running from Barefoot Running: An Evolutionary Medical Perspective. Exerc Sport Sci Rev. 2012;40(2):63-72. DOI: 10.1097/JES.0b013e31824ab210.

8. Davis IS. The re-emergence of the minimal running shoe. J Orthop Sports Phys Ther. 2014;44(10):775-84. DOI: 10.2519/jospt.2014.5521.

9. Lieberman DE, Venkadesan M, Werbel WA, Daoud AI, D'Andrea S, Davis IS, et al. Foot strike patterns and collision forces in habitually barefoot versus shod runners. Nature. 2010;463(7280):531-5. DOI: 10.1038/nature08723.

10. Nigg BM, Baltich J, Hoerzer S, Enders H. Running shoes and running injuries: mythbusting and a proposal for two new paradigms: «preferred movement path» and «comfort filter». Br J Sports Med. 2015;49(20):1290-4. DOI: 10.1136/bjsports-2015-095054.

11. World Rankings | Men's Marathon (Half Marathon-25km-30km) [Internet]. [citado 19 de enero de 2020]. Disponible en: https://www.worldathletics.org/world-rankings/marathon/men

12. Mündermann A, Nigg BM, Stefanyshyn DJ, Humble RN. Development of a reliable method to assess footwear comfort during running. Gait Posture. 2002;16(1):38-45. DOI: 0.1016/s0966-6362(01)00197-7.

13. Esculier JF, Dubois B, Dionne CE, Leblond J, Roy JS. A consensus definition and rating scale for minimalist shoes. J Foot Ankle Res. 2015;8:42. DOI: 10.1186/s13047-015-0094-5.

14. Ramsey CA, Ribeiro DC, Lamb P, Sole CC, Sole G. Reliability of the footwear total asymmetry score tool. Footwear Sci.;10(2):119-28. DOI: 10.1080/19424280.2018.1478888.

15. Barton CJ, Bonanno D, Menz HB. Development and evaluation of a tool for the assessment of footwear characteristics. J Foot Ankle Res. 2009;2:10. DOI: 10.1186/1757-1146-2-10.

16. Ramsey CA, Lamb P, Kaur M, Baxter GD, Ribeiro DC. How are running shoes assessed? A systematic review of characteristics and measurement tools used to describe running footwear. J Sports Sci. 2019;37(14):1617-29. DOI: 10.1080/02640414.2019.1578449.

17. Ryan M, Elashi M, Newsham-West R, Taunton J. Examining injury risk and pain perception in runners using minimalist footwear. Br J Sports Med. 2014;48(16):1257-62. DOI: 10.1136/bjsports-2012-092061.

18. Hébert-Losier K, Newsham-West RJ, Schneiders AG, Sullivan SJ. Raising the standards of the calf-raise test: a systematic review. J Sci Med Sport. 2009;12(6):594-602. DOI: 10.1016/j.jsams.2008.12.628.

19. Möller M, Lind K, Styf J, Karlsson J. The reliability of isokinetic testing of the ankle joint and a heel-raise test for endurance. Knee Surg Sports Traumatol Arthrosc. 2005;13(1):60-71. DOI: 10.1007/s00167-003-0441-0.

20. Sole CC, Milosavljevic S, Sole G, Sullivan SJ. Exploring a model of asymmetric shoe wear on lower limb performance. Phys Ther Sport. 2010;11(2):60-5. DOI: 10.1016/j.ptsp.2010.02.002.

21. Weeks BK, Carty CP, Horan SA. Kinematic predictors of single-leg squat performance: a comparison of experienced physiotherapists and student physiotherapists. BMC Musculoskelet Disord. 2012;13:207. DOI: 10.1186/1471-2474-13-207.

22. Newton RU, Gerber A, Nimphius S, Shim JK, Doan BK, Robertson $M$, et al. Determination of functional strength imbalance of the lower extremities. J Strength Cond Res. 2006;20(4):971-7. DOI: 10.1519/R-5050501x.1.

23. Shanthikumar S, Low Z, Falvey E, McCrory P, Franklyn-Miller A. The effect of gait velocity on calcaneal balance at heel strike; Implications for orthotic prescription in injury prevention. Gait Posture. 2010;31(1):912. DOI: 10.1016/j.gaitpost.2009.08.003.

24. Hunter I, Smith GA. Preferred and optimal stride frequency, stiffness and economy: changes with fatigue during a 1-h high-intensity run. Eur J Appl Physiol. 2007;100(6):653-61. DOI: 10.1007/s00421-007-0456-1.

25. Hayes $P$, Caplan N. Foot strike patterns and ground contact times during high-calibre middle-distance races. J Sports Sci. 2012;30(12):1275-83. DOI: 10.1080/02640414.2012.707326.

26. Tam N, Wilson JLA, Noakes TD, Tucker R. Barefoot running: an evaluation of current hypothesis, future research and clinical applications. $\mathrm{Br}$ J Sports Med. 2014;48(5):349-55. DOI: 10.1136/bjsports-2013-092404.

27. Kasmer ME, Liu XC, Roberts KG, Valadao JM. Foot-strike pattern and performance in a marathon. Int J Sports Physiol Perform. 2013;8(3):28692. DOI: 10.1123/ijspp.8.3.286.

28. Hasegawa $\mathrm{H}$, Yamauchi T, Kraemer WJ. Foot strike patterns of runners at the 15-km point during an elite-level half marathon. J Strength Cond Res. 2007;21(3):888-93. DOI: 10.1519/R-22096.1.

29. Nigg BM, Segesser B. Biomechanical and orthopedic concepts in sport shoe construction. Med Sci Sports Exerc. 1992;24(5):595-602.

30. Theisen D, Malisoux L, Genin J, Delattre N, Seil R, Urhausen A. Influence of midsole hardness of standard cushioned shoes on running-related injury risk. Br J Sports Med. 2014;48(5):371-6. DOI: 10.1136/ bjsports-2013-092613.

31. Pollard CD, Ter Har JA, Hannigan JJ, Norcross MF. Influence of Maximal Running Shoes on Biomechanics Before and After a $5 \mathrm{~K}$ Run. Orthop J Sports Med. 2018;6(6):2325967118775720. DOI: 10.1177/2325967118775720.

32. Baltich J, Maurer C, Nigg BM. Increased vertical impact forces and altered running mechanics with softer midsole shoes. PLoS One. 2015;10(4):e0125196. DOI: 10.1371/journal.pone.0125196.

33. Miller RH. Joint Loading in Runners Does Not Initiate Knee Osteoarthritis. Exerc Sport Sci Rev. 2017;45(2):87-95. DOI: 10.1249/ JES.0000000000000105.

34. Kulmala JP, Kosonen J, Nurminen J, Avela J. Running in highly cushioned shoes increases leg stiffness and amplifies impact loading. Sci Rep. 2018;8(1):17496. DOI: 10.1038/s41598-018-35980-6..

35. Nigg BM. The role of impact forces and foot pronation: a new paradigm. Clin J Sport Med. 2001; DOI: 10.1097/00042752-200101000-00002.

36. Kong PW, Candelaria NG, Smith DR. Running in new and worn shoes: a comparison of three types of cushioning footwear. $\mathrm{Br} J$ Sports Med. 2009;43(10):745-9. DOI: 10.1136/bjsm.2008.047761.

37. Richards CE, Magin PJ, Callister R. Is your prescription of distance running shoes evidence-based? Br J Sports Med. 2009;43(3):159-62. DOI: 10.1136/bjsm.2008.046680. 
38. Nielsen RO, Buist I, Parner ET, Nohr EA, Sørensen H, Lind M, et al. Foot pronation is not associated with increased injury risk in novice runners wearing a neutral shoe: a 1-year prospective cohort study. $\mathrm{Br} J$ Sports Med. 2014;48(6):440-7. DOI: 10.1136/bjsports-2013-092202.

39. Ryan MB, Valiant GA, McDonald K, Taunton JE. The effect of three different levels of footwear stability on pain outcomes in women runners: a randomised control trial. Br J Sports Med. 2011;45(9):715-21. DOI: 10.1136/bjsm.2009.069849.

40. Sole CC, Milosavljevic S, Sole G, Sullivan SJ. Patterns of mediolateral asymmetry in worn footwear. Footwear Sci. 20014;6(3):177-92. DOI: 10.1080/19424280.2014.913694.

41. Radzimski AO, Mündermann A, Sole G. Effect of footwear on the external knee adduction moment - A systematic review. Knee. 2012;19(3):16375. DOI: 10.1016/j.knee.2011.05.013.

42. Kerrigan DC, Franz JR, Keenan GS, Dicharry J, Della Croce U, Wilder RP. The effect of running shoes on lower extremity joint torques. PM R. 2009; 1(12):1058-63. DOI: 10.1016/j.pmrj.2009.09.011.

43. Leveau B. Normal and Abnormal Function of the Foot: Clinical Biomechanics, vol 2. Phys Ther. 1979;59(3):352-2.

44. Uceta MEF, Huerta JP, Perdiguero JH, Ignasio NA. Efecto de las Ortesis Funcionales Sobre la Posición Frontal en Estática de Calcáneo y Tibia. Podol Clínica. 2010;11(1):18-25.

45. Eng JJ, Pierrynowski MR. The effect of soft foot orthotics on three-dimensional lower-limb kinematics during walking and running. Phys Ther. 1994;74(9):836-44. DOI: 10.1093/ptj/74.9.836.

46. Stacoff A, Nigg BM, Reinschmidt C, van den Bogert AJ, Lundberg A. Tibiocalcaneal kinematics of barefoot versus shod running. J Biomech. 2000;33(11):1387-95. DOI: 10.1016/s0021-9290(00)00116-0.

47. Nawoczenski DA, Cook TM, Saltzman CL. The effect of foot orthotics on three-dimensional kinematics of the leg and rearfoot during running. J Orthop Sports Phys Ther. 1995;21(6):317-27. DOI: 10.2519/ jospt. 1995.21.6.317.

48. Luo G, Stergiou P, Worobets J, Nigg B, Stefanyshyn D. Improved footwear comfort reduces oxygen consumption during running. Footwear Sci. 2009;1(1):25-9. DOI: 10.1080/19424280902993001.

49. Mündermann A, Stefanyshyn D, Nigg B. Relationship between footwear comfort of shoe inserts and anthropometric and sensory factors. Med Sci Sports Exerc. 2001;33(11):1939-45. DOI: 10.1097/00005768200111000-00021.
50. Basford JR, Smith MA. Shoe insoles in the workplace. Orthopedics. 1988; 11(2):285-8.

51. Mohr M, Meyer C, Nigg S, Nigg B. The relationship between footwear comfort and variability of running kinematics. Footwear Sci. 2017;9(sup1):S45-7. DOI: 10.1080/19424280.2017.1314329.

52. Che H, Nigg BM, de Koning J. Relationship between plantar pressure distribution under the foot and insole comfort. Clin Biomech (Bristol, Avon). 1994;9(6):335-41. DOI: 10.1016/0268-0033(94)90062-0.

53. Nigg BM, Nurse MA, Stefanyshyn DJ. Shoe inserts and orthotics for sport and physical activities. Med Sci Sports Exerc. 1999;31(7 Suppl):S421-8. DOI: 10.1097/00005768-199907001-00003.

54. Collins N, Crossley K, Beller E, Darnell R, McPoil T, Vicenzino B. Foot orthoses and physiotherapy in the treatment of patellofemoral pain syndrome: randomised clinical trial. BMJ. 2008;337:a1735. DOI: 10.1136/bmj.a1735.

55. Collins NJ, Hinman RS, Menz HB, Crossley KM. Immediate effects of foot orthoses on pain during functional tasks in people with patellofemoral osteoarthritis: A cross-over, proof-of-concept study. Knee. 2017;24(1):76-81. DOI: 10.1016/j.knee.2016.09.016.

56. House CM, Waterworth C, Allsopp AJ, Dixon SJ. The influence of simulated wear upon the ability of insoles to reduce peak pressures during running when wearing military boots. Gait Posture. 2002;16(3):297303. DOI: 10.1016/s0966-6362(02)00021-8.

57. Saito S, Muraki S, Tochihara Y. Effects of worn-out soles on lower limb stability, shock absorption and energy cost during prolonged walking. J Physiol Anthropol. 2007;26(5):521-6. DOI: 10.2114/jpa2.26.521.

58. Hagen M, Hennig EM. Effects of different shoe-lacing patterns on the biomechanics of running shoes. J Sports Sci. 2009;27(3):267-75. DOI: 10.1080/02640410802482425.

59. Hong Y, Wang L, LiJX, Zhou JH. Changes in running mechanics using conventional shoelace versus elastic shoe cover. J Sports Sci. 2011;29(4):3739. DOI: 10.1080/02640414.2010.534805.

60. Hagen M, Hömme AK, Umlauf T, Hennig EM. Effects of different shoe-lacing patterns on dorsal pressure distribution during running and perceived comfort. Res Sports Med. 2010;18(3):176-87. DOI: 10.1080/15438627.2010.490180.

61. Malisoux L, Ramesh J, Mann R, Seil R, Urhausen A, Theisen D. Can parallel use of different running shoes decrease running-related injury risk? Scand J Med Sci Sports. 2015;25(1):110-5. DOI: 10.1111/sms. 12154. 